\title{
Two-dimensional electronic spectroscopy with birefringent wedges
}

Cite as: Rev. Sci. Instrum. 85, 123107 (2014); https://doi.org/10.1063/1.4902938

Submitted: 23 September 2014 . Accepted: 18 November 2014 . Published Online: 09 December 2014

Julien Réhault $\mathbb{D}$, Margherita Maiuri, Aurelio Oriana, and Giulio Cerullo
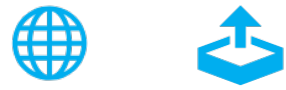

\section{ARTICLES YOU MAY BE INTERESTED IN}

Ultrafast optical parametric amplifiers

Review of Scientific Instruments 74, 1 (2003); https://doi.org/10.1063/1.1523642

Phase-stabilized two-dimensional electronic spectroscopy

The Journal of Chemical Physics 121, 4221 (2004); https://doi.org/10.1063/1.1776112

Fluorescence-detected two-dimensional electronic coherence spectroscopy by acousto-optic phase modulation

The Journal of Chemical Physics 127, 214307 (2007); https://doi.org/10.1063/1.2800560

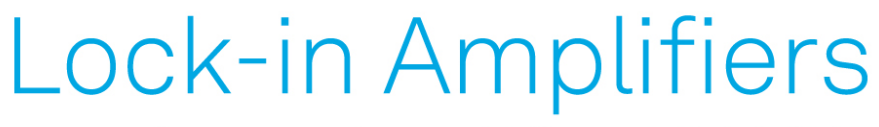
... and more, from DC to $600 \mathrm{MHz}$

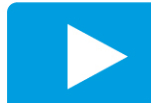

Watch 


\title{
Two-dimensional electronic spectroscopy with birefringent wedges
}

\author{
Julien Réhault, Margherita Maiuri, Aurelio Oriana, and Giulio Cerullo \\ IFN-CNR, Dipartimento di Fisica, Politecnico di Milano, Piazza Leonardo da Vinci 32, 20133 Milano, Italy
}

(Received 23 September 2014; accepted 18 November 2014; published online 9 December 2014)

\begin{abstract}
We present a simple experimental setup for performing two-dimensional (2D) electronic spectroscopy in the partially collinear pump-probe geometry. The setup uses a sequence of birefringent wedges to create and delay a pair of phase-locked, collinear pump pulses, with extremely high phase stability and reproducibility. Continuous delay scanning is possible without any active stabilization or position tracking, and allows to record rapidly and easily 2D spectra. The setup works over a broad spectral range from the ultraviolet to the near-IR, it is compatible with few-optical-cycle pulses and can be easily reconfigured to two-colour operation. A simple method for scattering suppression is also introduced. As a proof of principle, we present degenerate and two-color 2D spectra of the light-harvesting complex 1 of purple bacteria. ( 2014 AIP Publishing LLC. [http://dx.doi.org/10.1063/1.4902938]
\end{abstract}

\section{INTRODUCTION}

Two-dimensional electronic spectroscopy (2DES) is a powerful technique for the study of structure and dynamics of multi-chromophore systems, measuring how the electronic states of different units within a complex molecular architecture interact with one another and transfer electronic excitations. ${ }^{1-7}$ It gives detailed insights into energy relaxation pathways by monitoring the coupling or the energy transfer between electronic states in time. 2DES in the visible was used to study photosynthetic light harvesting complexes, ${ }^{8-11}$ molecular aggregates, ${ }^{12,13}$ and semiconductor nanostructures ${ }^{14-16}$ and its extension to the UV range is extremely promising for the study of biomolecules such as DNA and proteins. ${ }^{17,18}$

2DES can be seen as an extension of ultrafast pumpprobe spectroscopy, with the extra capability of resolving the pump frequency dependence of the transient absorption signals. In pump-probe spectroscopy, a short and broadband pulse is used to excite the sample. Due to the Fourier limit of the time-bandwidth product, a high time resolution requires a broad bandwidth of the excitation pulse. Such broadband pulse can usually access several excited states of the sample, each of which has its own photophysics or photochemistry. The resulting transient absorption spectra consist in a superposition of different excited states corresponding to the photoexcited ground state transitions. To enhance selectivity, it is possible to narrow the pump pulse bandwidth, ${ }^{19}$ but this comes at the cost of a reduced time resolution. Reconstruction of $2 \mathrm{D}$ spectra can be performed by tuning the pump pulse frequency, and this approach is referred to as frequency-domain 2D spectroscopy. The first experimental 2D vibrational spectra in the mid-infrared were acquired with this technique, using a Fabry-Perot interferometer as a tunable narrow-bandwidth filter ${ }^{20}$; this principle was also recently extended to the visible ${ }^{21}$ and ultraviolet spectral ranges. ${ }^{22}$ In the frequency-domain approach, each line in the pump frequency axis is a pump-probe spectrum acquired with narrowband excitation.

With time-domain 2D spectroscopy, both temporal and spectral resolution are preserved. The principle of time- domain 2DES is illustrated in Fig. 1: the system under study is excited by three consecutive pulses, with controllable relative delays $t_{1}$ and $t_{2}$. This pulse sequence builds up a macroscopic third-order nonlinear polarization in the medium that emits a field, following pulse 3 . This field is fully measured in amplitude and phase by interference with an auxiliary pulse, known as the local oscillator (LO), phase-coherent with pulse 3 . By Fourier transforming with respect to $t_{1}$ and $t_{3}$ for a fixed value of the waiting time $\mathrm{t}_{2}$, one derives the $2 \mathrm{D}$ spectrum as a function of pump frequency $\omega_{1}$ and probe frequency $\omega_{3}$. Typically, the Fourier transform with respect to $t_{3}$ is performed by a spectrometer (see Fig. 2), while that with respect to $t_{1}$ is performed numerically. When the pump axis is vertical, and the probe axis horizontal, each horizontal line of the 2D map can be interpreted as a pump-probe spectrum at a specific pump frequency. The integral of the 2D map along the pump frequency axis gives the traditional pump-probe spectrum and can be used to check consistency of the results and correct the phase used in the Fourier transform. To acquire time-domain 2D spectra, two different configurations can be used: the heterodyne detected three-pulse photon echo ${ }^{23-25}$ and the partially collinear pump-probe geometry. ${ }^{26-28}$

Heterodyne-detected three-pulse photon echo exploits the non-collinear interaction geometry between the three driving pulses to emit the four-wave-mixing echo signal in a background-free direction, dictated by phase-matching (Fig. 2). The echo signal is fully resolved in amplitude and phase using spectral interferometry with a fourth heterodyning pulse (the LO). The heterodyned signal is detected with a spectrometer, which optically Fourier transforms the signal generating the $\omega_{\text {probe }}$ (or $\omega_{3}$ ) axis; a numerical Fourier transform with respect to $t_{1}$ is used to generate the $\omega_{\text {pump }}$ (or $\omega_{1}$ ) axis.

The three-pulse photon echo is the more flexible and sensitive approach, at the cost of a more complicated experimental setup. ${ }^{24,29}$ The four pulses (pulses $1-3$ and the LO) are aligned in a boxcar geometry before impinging on the sample. Interferometric stability is required between pulses 1 and 2 and between pulse 3 and the LO. In most implementations of 2DES, phase-locking has been achieved by using as beam splitters diffractive optics. Alternatively, conventional 


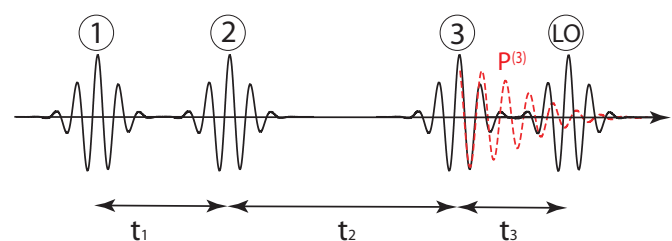

FIG. 1. Principle of heterodyne-detected time-domain 2D spectroscopy.

delay lines with active path-length stabilization can be used, ${ }^{30}$ at the expense of a significant complication of the experimental setup. Recently, an implementation of 2DES that employs only conventional beam splitters and mirrors has been demonstrated. ${ }^{31}$ This approach uses delay lines that handle the four pulses always in pairs, so that the two pulses from any pair induce opposite phase shifts in the interference signal. The three-pulse photon echo method allows to record, depending on the time ordering of the two pump pulses, the so-called "rephasing" and "non-rephasing" spectra. ${ }^{7}$ The absorptive lineshape, most commonly used for interpretation of the spectra, is the sum of these two contributions and requires to measure them independently. The polarization and intensity of each pulse can be fully controlled, allowing to access different response pathways.

The partially collinear pump-probe geometry requires two phase-locked collinear pump pulses and a non-collinear probe pulse, which is dispersed on a spectrometer (Fig. 2(b)). The probe pulse has the dual purpose of generating the nonlinear polarization and heterodyning it (self-heterodyning configuration). Advantages of this configuration are its simplicity and the fact that it automatically measures absorptive spectra, which are the most direct to interpret. An additional important advantage of this geometry is that it easily lends itself to the extension to two-color 2DES.

(a)

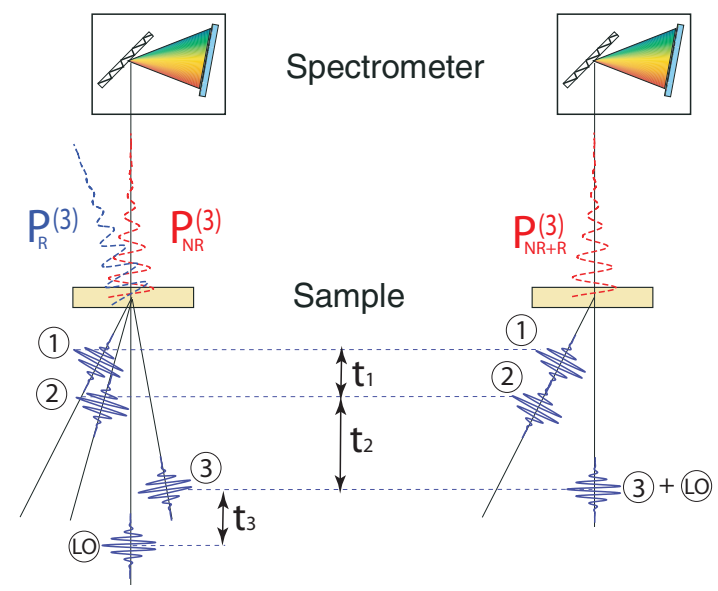

FIG. 2. (a) Heterodyne detected three-pulse photon echo geometry, where the three excitation pulses are non-collinear. Rephasing $\left(\mathrm{P}_{R}^{(3)}\right)$ and nonrephasing $\left(\mathrm{P}_{N R}^{(3)}\right)$ signals are emitted in a new direction, and are heterodyned by a fourth pulse (LO) on the detector $\left(\mathrm{P}_{R}^{(3)}\right.$ and $\mathrm{P}_{N R}^{(3)}$ signals can be inverted by inverting the time ordering of pulses 1 and 2 and recorded them in the same LO direction). (b) In the pump-probe geometry, the rephasing and nonrephasing signals are emitted in the same direction as the probe which also heterodynes them on the detector.
The partially collinear pump-probe geometry is simpler in principle, as it requires the alignment of only two beams on the sample. It is less flexible and less sensitive, as the different field interactions are not independent, and because probe intensity is limited by the dynamic range of the detector (in three-pulse photon echo, the probe pulse can be arbitrarily intense as it does not hit the detector, and only the LO intensity is limited by the detector dynamic range). Flexibility on polarization and intensity can be re-gained using strategies involving polarizers in the probe beam before and after sample. ${ }^{32-34}$ The main advantage of the partially collinear geometry is the fact that it is a straightforward extension of standard pump-probe, in which a pulse-pair generator is inserted in the pathway of the pump beam. This should allow many research groups to easily upgrade their pumpprobe spectrometer to 2DES operation, as recently proposed by Shim and Zanni. ${ }^{35}$ The remaining technical difficulty is the generation of a collinear phase-locked pump-pulse pair. Balanced Michelson/Mach-Zehnder interferometers with active stabilization or inline measurement of the path-length difference $^{36}$ can be used in the IR. However, the stabilization becomes more demanding when moving to the visible or the UV range. Alternatively, phase-locked pulse pairs can be produced using pulse shapers, which inherently provide interferometric stability due to the common path of the two pulses. Sinusoidal modulation of both amplitude and phase is required for the generation of a pulse pair. Several solutions, including liquid crystal spatial light modulators, ${ }^{37}$ acoustooptic modulators, ${ }^{28}$ and acousto-optic programmable dispersive filters ${ }^{38}$ have been proposed. However, pulse shapers considerably increase the complexity and the cost of the experimental apparatus, have sometimes low throughput and work only in a limited spectral range.

We have recently introduced a novel passive optical device, which we called TWINS (Translating-Wedge- Based Identical Pulses eNcoding System), solving in a simple, compact and cost-effective way the problem of building a collinear delay line with interferometric stability. ${ }^{39}$ TWINS uses birefringence to impose an arbitrary delay on two orthogonal polarization components by continuously varying the material thickness. TWINS has already been successfully implemented in the visible ${ }^{39}$ and the $\mathrm{IR}^{40}$ ranges demonstrating excellent phase stability and reproducibility. In this paper, we describe in detail our 2DES setup, working in the partially collinear pump-probe geometry and using TWINS to generate the phase-locked pump pulses. Its characteristics are the simplicity, its broad acceptance bandwidth, easily allowing two-color operation, and the extreme accuracy in the $t_{1}$ delay, allowing continuous scan without any stabilization or tracking. We are confident that our approach, by removing technological barriers, will stimulate many research groups to adopt 2DES to solve a variety of problems in physics, chemistry, and biology.

\section{TWINS DESIGN AND ALIGNMENT}

\section{A. TWINS principle}

TWINS is a compact, passive optical device which exploits birefringence to create two collinear, phase-locked 
(a)

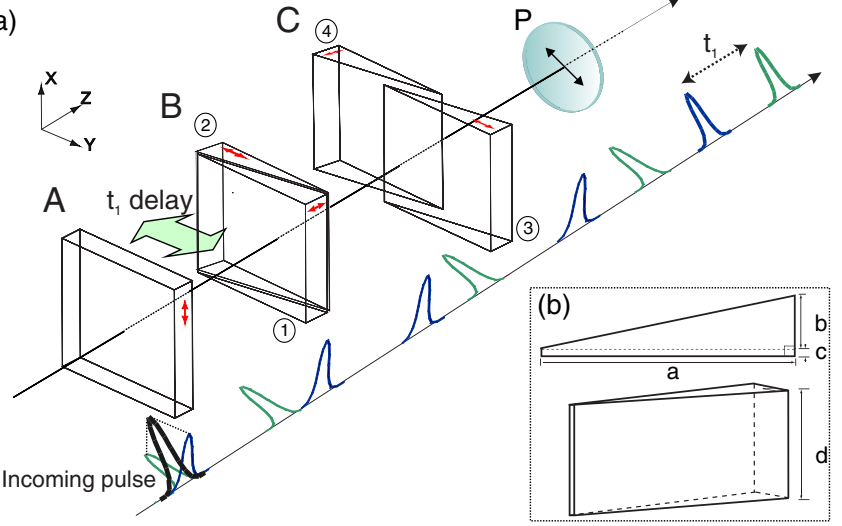

FIG. 3. (a) Scheme of the TWINS setup. Block A creates a constant "negative" delay $t_{1}$ between two orthogonally polarized pulses. Block B scans the delay $\mathrm{t}_{1}$ between the pump pulses towards positive time. Block $\mathrm{C}$ corrects angular dispersion, front tilt and allows to tune dispersion accurately. Polarizer $\mathrm{P}$ projects the two pump pulses to a common polarization direction. Small arrows indicate the direction of the optical axis of the birefringent plates. The big arrow indicates the movement direction of block B.(b) wedge design, $\mathrm{a}=25 \mathrm{~mm}, \mathrm{~b}=3.1 \mathrm{~mm}, \mathrm{c}=0.5 \mathrm{~mm}$, and $\mathrm{d}=20 \mathrm{~mm}$.

replicas of an input pulse. If a pulse enters a birefringent plate with polarization at $45^{\circ}$ with respect to the ordinary and extraordinary axes, it splits into two perpendicularly polarized pulses of the same intensity, propagating with different group velocities. If the thickness of the birefringent plate is varied, which can be achieved by replacing the plate with a pair of wedges, then the delay between these two pulses will also be varied. Since the two pulse replicas follow the same optical path, they are phase-locked with very high stability and their delay can be controlled, with extreme precision and reproducibility, by changing the insertion of the birefringent wedges.

To perform 2D spectroscopy with TWINS, two more requirements must be fulfilled: (i) one must be able to access the delay zero between the two pump pulses, and (ii) pump pulse 2 and pulse 3 (the probe) need to maintain a constant delay during the $t_{1}$ scan. The first requirement is fulfilled by block A in Fig. 3(a), whose constant thickness compensates the overall minimum delay introduced by blocks B and C. It introduces a constant delay for the Y-polarized pulse relative to the X-polarized one (see Fig. 3(a) for a definition X,Y,Z orientations). The second requirement is satisfied by the pair of wedges in block B. Due to the different orientations of the optical axes in the two wedges, the X-polarized pulse sees a constant optical path even when this block is moved into the beam, as the two wedges are geometrically identical. The Y-polarized pulse experiences a change in optical thickness, while block B is moved into the beam as depicted by the green arrow in Fig. 3(a). When using a negative birefringence material $\left(\mathrm{n}_{e}<\mathrm{n}_{o}\right)$, increasing thickness of the birefringent wedge (wedge 2) decreases the optical path for the Y-polarized pulse, and this pulse arrives earlier than the X-polarized one. After exiting block B, the pulses are slightly non-collinear and front-tilted, because they experience different refractions at each interface, and because the phase front experiences different thickness of birefringent material. This is corrected by the static block $\mathrm{C}$, consisting of two wedges identical to those in block B, and placed as close as possible to block B (typically less than $5 \mathrm{~mm}$ ) to minimize the lateral displacement between the pump pulses, which is negligible under our experimental conditions. ${ }^{40}$ The insertion of the wedges of block $\mathrm{C}$ into the beam can be kept small and is also used to finely tune the dispersion introduced by the overall sequence (see Sec. III C). A polarizer placed at the end of the wedge sequence projects, if so required by the experimental configuration, the two pulses to a common polarization direction and can be removed if two orthogonally polarized pump pulses are required.

\section{B. TWINS design and mounting}

We used $\alpha-\mathrm{BaB}_{2} \mathrm{O}_{4}(\alpha-\mathrm{BBO})$ as a negative uniaxial birefringent material for the wedges. We distinguish Y-cut, $\mathrm{X}$ cut, and Z-cut wedges by the orientation of their optical axis with respect to the coordinate system displayed in Fig. 3(a). The wedges are designed according to the drawing shown in Fig. 3(b); one pair is Z-cut and the other is Y-cut (it could as well be X-cut). The sizes depend on the maximum delay one wants to achieve, the important parameter being the length of the $\mathrm{b}$ side. The additional thickness $\mathrm{c}$ at the apex is in principle not required, but is necessary for production. The wedge pairs were purchased from FOCtek Photonics Inc. The quality of the wedges is not perfect and we observed that while two wedges of the same pair (Z-cut and Y-cut) were undistinguishable, the apex angle from one pair to the other was slightly different, most probably because of processing tolerances. Also the optical axis is not perfectly along $\mathrm{Z}$ or $\mathrm{Y}$. This has an impact on the proper functioning of the TWINS, but can be easily corrected following the alignment procedure detailed in Sec. II C.

\section{TWINS alignment procedure}

Each wedge is placed on a kinematic mount that allows rotation in the $(\mathrm{X}, \mathrm{Z})$ and $(\mathrm{Y}, \mathrm{Z})$ planes. Wedge 3 is mounted on a goniometer and has the additional degree of freedom of rotation in the $(\mathrm{X}, \mathrm{Y})$ plane. Additionally, wedges 1 and 4 are mounted on translation stages along $\mathrm{Z}$ direction to minimize the distance separating them from the next wedge, and wedges 3 and 4 are mounted on translation stages along the $Y$ direction that allow to change their insertion into the beam. The proper alignment of the TWINS device is a prerequisite to perform 2DES. The two main important conditions are: (a) a constant delay of pulse 2 with respect to pulse 3 (the probe pulse, so that $t_{2}$ is constant during a $t_{1}$ scan) and (b) a good contrast of the spectral interference fringes between pulses 1 and 2 . Both can be optimized with proper alignment.

The constant delay of arrival of pulse 2 in an absolute frame of reference would be in principle guaranteed by the constant thickness of block $B$ while scanning $t_{1}$. However, since the Z- and Y-cut wedges are not absolutely identical, the arrival time of pulse 2 can change during the scan of block B. This can be easily measured by performing spectral interferometry between pulse 2 and the probe. To this purpose, we align the output polarizer of the TWINS to transmit only the vertical component (pulse 2) and look through a $25 \mu \mathrm{m}$ 
pinhole at the spectral interference fringes between pulse 2 and the probe into the spectrometer. The fringes should not move during the scan of $t_{1}$, but if they do, we can correct the effective thickness of block $B$ by rotating the angle of wedge 2 in the $(\mathrm{Z}, \mathrm{X})$ plane, until the fringes are stationary during a full scan of $t_{1}$.

The alignment and contrast optimization of the wedges is performed by using a large collimated $\mathrm{He}-\mathrm{Ne}$ laser beam. First, we place block B into the beam. This way, the two orthogonally polarized components exit with different angles and are spatially separated after a few centimetres. We then set the input polarization to $45^{\circ}$ by balancing the intensity of the two components. We insert the wedges of block $\mathrm{C}$ into the beam (with the translation stages, they can be easily inserted or removed). The output polarizer is then placed at $\pm 45^{\circ}$ relative to the first one. The output beam normally has spatial fringes whose density should be minimized by using all the rotational degrees of freedom. Using this procedure, we typically achieve a contrast of a few thousands between bright and dark fringes with a $1 \mathrm{~mm} \mathrm{He}-\mathrm{Ne}$ beam.

\section{TWINS advantages and characteristics}

The main advantage of TWINS is its capability to generate a pair of phase-locked collinear pump pulses with delay which can be controlled to extreme precision even with standard motorized translation stages and without any feedback loop for active position control. ${ }^{41}$ One can calculate a gear ratio which corresponds to the ratio between the translation required to introduce a certain delay with the TWINS and the translation required with a standard interferometer in free space with a retroreflector. This gear ratio for $\alpha$-BBO at $500 \mathrm{~nm}$ and wedges with an apex angle of $7^{\circ}$ is $\approx 60$. In fact, a motor resolution of $0.01 \mu \mathrm{m}$ translates into a precision in delay of $\approx 0.5$ attoseconds between the pulses. This extreme precision in delay control, combined with the phase-locking property of the pair of pulses exiting the TWINS setup, open the way to fast scanning without the use of any tracking schemes, as would be required in other 2D setups using conventional interferometers. ${ }^{36}$ The constant velocity of the motor insures even time spacing between subsequent pulses coming from our $1 \mathrm{kHz}$ repetition rate laser. It is also possible to combine forward and backward scans to double the acquisition rate. The Sellmeier equations allow us to calculate the maximum group delays achievable for a given thickness of the wedges, which are summarized in Table I for $\alpha$-BBO at different wavelengths from near-IR to UV. This delay is given by $\Delta t_{\max }=\mathrm{GVM} \mathrm{d}$ where GVM is the group velocity mismatch $\left(G V M=1 / v_{g o}-1 / v_{g e}\right)$ between ordinary $\left(v_{g o}\right)$ and extraordinary $\left(v_{g e}\right)$ polarizations and $\mathrm{d}$ is the thickness of birefringent material. The maximum obtainable spectral resolution $\Delta v$ can be calculated through the relation: ${ }^{42}$

$$
\Delta v=\frac{1}{\Delta t_{\max }} .
$$

With our wedge designs we can achieve group delay in excess of $1 \mathrm{ps}$ in the visible, which are more than sufficient for the typical linewidths of electronic transitions.
TABLE I. $\alpha$-BBO refractive indices $\mathrm{n}_{o}$ and $\mathrm{n}_{e}$, birefringence $\Delta \mathrm{n}$, Group velocity mismatch (GVM), and group velocity dispersion for ordinary and extraordinary axis (GVDo and GVDe).

\begin{tabular}{lcccccc}
\hline \hline $\begin{array}{l}\lambda \\
(\mathrm{nm})\end{array}$ & $\mathrm{n}_{o}$ & $\mathrm{n}_{e}$ & $\Delta \mathrm{n}$ & $\begin{array}{c}\mathrm{GVM} \\
(\mathrm{fs} / \mathrm{mm})\end{array}$ & $\begin{array}{c}\text { GVDo } \\
\left(\mathrm{fs}^{2} / \mathrm{mm}\right)\end{array}$ & $\begin{array}{c}\text { GVDe } \\
\left(\mathrm{fs}^{2} / \mathrm{mm}\right)\end{array}$ \\
\hline 1100 & 1.6573 & 1.5374 & 0.1199 & 402.5 & 37.8 & 16.6 \\
900 & 1.6623 & 1.5410 & 0.1203 & 411.0 & 60.9 & 37.2 \\
700 & 1.6674 & 1.5460 & 0.1215 & 427.1 & 91.3 & 61.0 \\
500 & 1.6807 & 1.5557 & 0.1250 & 468.2 & 149.1 & 102 \\
300 & 1.7342 & 1.5928 & 0.1414 & 672.6 & 391.5 & 260.7 \\
\hline \hline
\end{tabular}

\section{2DES SPECTROMETER USING TWINS}

\section{A. Experimental setup}

Our 2DES setup starts with a commercial amplified Ti:sapphire laser system (Libra, Coherent) delivering $4 \mathrm{~mJ}$, $100 \mathrm{fs}$ pulses at $800 \mathrm{~nm}$, and $1 \mathrm{kHz}$ repetition rate. The system pumps three synchronized non-collinear optical amplifiers (NOPAs) which feed the 2DES setup. The first NOPA (NOPA 1) delivers visible pulses with a spectrum spanning between $500 \mathrm{~nm}$ and $750 \mathrm{~nm}$, compressed to sub-10-fs duration by multiple bounces on custom-designed double-chirped mirrors $^{43}$ (DCMs, Laser Optiks). The second NOPA (NOPA 2) delivers near-infrared (NIR) pulses with a spectrum spanning between $850 \mathrm{~nm}$ and $1100 \mathrm{~nm}$, compressed to sub-15-fs duration by a double pass in a Brewster-cut fused silica prism pair. ${ }^{44,45}$ The third NOPA (NOPA 3), built according to the design of Ref. 46, delivers pulses in the 700-1000 nm range, compressed to sub-10-fs duration by multiple bounces on DCMs. The layout of the 2DES setup is presented in Fig. 4. Briefly, for degenerate 2DES, pump and probe are separated by an inconel beamsplitter (in two-colour configuration the two beams come from different NOPAs). The pair of pump pulses is generated by the TWINS setup, assembled according to Fig. 2. Block B is mounted on a miniature translation stage (M-112.2DG Physik Instrumente $\mathrm{GmbH}$ ) with resolution better than $0.01 \mu \mathrm{m}$ and a maximum speed of $2 \mathrm{~mm} / \mathrm{s}$. The probe pulses are delayed by $t_{2}$ with motor $\mathrm{M}_{1}$ (M-112.2DG Physik Instrumente $\mathrm{GmbH}$ ), and focused on the sample by spherical mirror SM (125 mm focal length) together with the pump pulses. The probe is collected by lens $\mathrm{L}_{1}$ and focused on the entrance slit of the spectrometer with lens $\mathrm{L}_{2}$. The spectrometer (Princeton Instruments, Acton Sp2150) is equipped with a CCD camera (Entwicklungsbro Stresing) reading out data at the full $1-\mathrm{kHz}$ repetition rate of the laser. ${ }^{47}$ Pinholes are placed in the probe beam after the sample to minimize scattered light from the pump. Pump pulses are prechirped with DCMs to compensate for the TWINS dispersion (see Sec. III C). Part of the pump beam is split off to monitor pump pulses interferences on a photodiode synchronized with acquisition of the probe spectra. The main part of the pump beam is focused on the sample.

\section{B. Calibration and phasing procedure}

To properly calibrate the pump frequency axis of the 2D spectra, we measure spectral interferometry on the pair of 


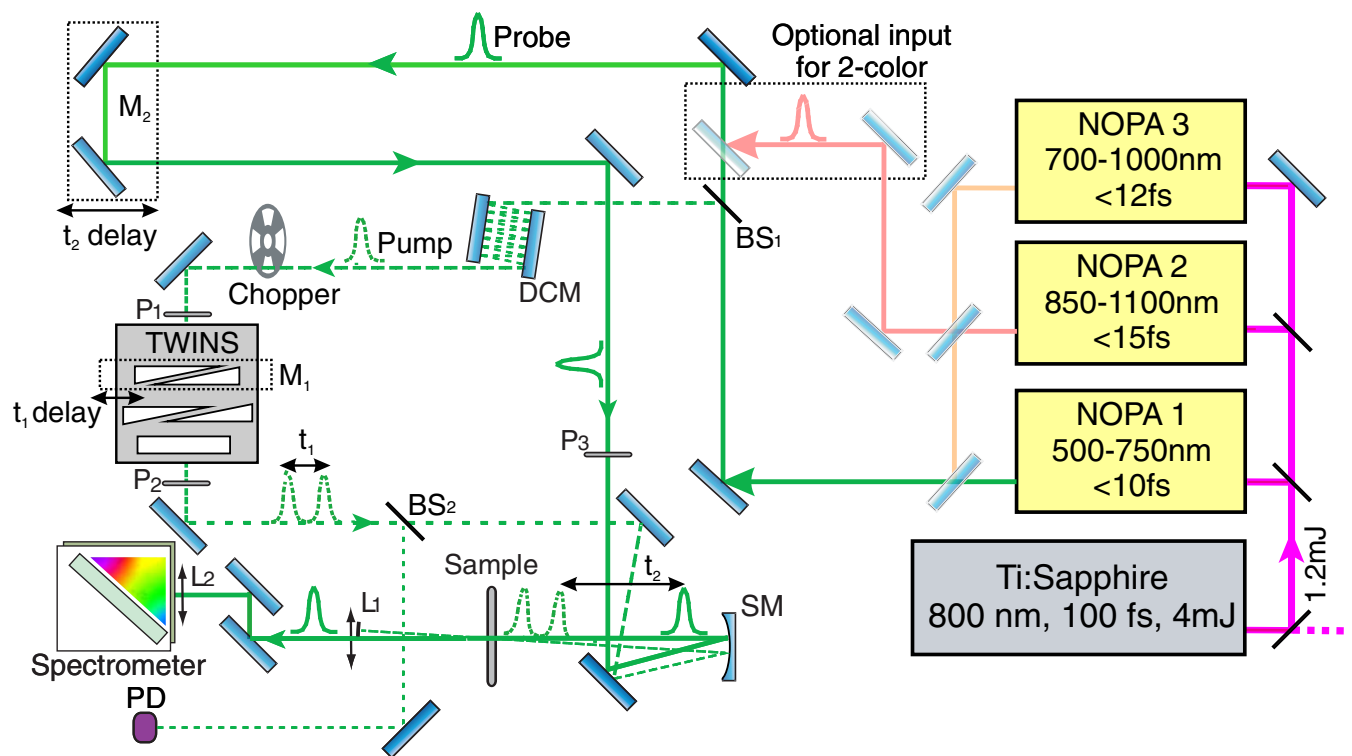

FIG. 4. Setup layout. $\mathrm{BS}_{1}$ : inconel beamsplitter, separating pump, and probe pulses. The continuous green line is the probe beam path, the dotted one is the pump beam path. $M_{1}$ and $M_{2}$ are the motors for the control of the coherence time $t_{1}$ and the population time delay $t_{2}$, respectively. $P_{1}$ and $P_{2}$ are the polarizers of the TWINS, at $45^{\circ}$ in the pump arm. $\mathrm{P}_{3}$ is the polarizer for the probe. SM: spherical mirror $(\mathrm{f}=125 \mathrm{~mm})$ to focus pump and probe pulses. $\mathrm{L}_{1}$ : collimating lens $(\mathrm{f}=100 \mathrm{~mm}) . \mathrm{L}_{2}$ : focusing lens $(\mathrm{f}=50 \mathrm{~mm})$. DCM: double chirped mirrors. $\mathrm{BS}_{2}$ : beamsplitter to select a small amount of pump pulse energy to monitor interferences on photodiode (PD).

pump pulses. We block the probe and place a $25 \mu \mathrm{m}$ pinhole at the sample position where we focus the pump beam. Part of the light diffracted by the pinhole is coupled into the spectrometer. We then record the interferograms between the two pump pulses by moving the motor continuously (as detailed in Sec. III E) with the same scan parameters used in the experiment. This leads to the two-dimensional map displayed in Fig. 5(a), which corresponds to a spectrally resolved linear autocorrelation of the two pump pulses. The intensity at each wavelength is plotted against the motor position during acquisition. The Fourier transform with respect to the motor position axis leads to a well defined peak whose frequency depends on the wavelength. The pump wavelength can then be calibrated ${ }^{39}$ (Fig. 5 (b)). This procedure, whose outcome depends only on the motor speed during the scan, takes a few seconds and is performed daily before a series of $2 \mathrm{DES}$ measurements.

To properly phase the $2 \mathrm{D}$ spectra, one needs to determine precisely the delay between the two pump pulses from the interferogram. This phasing procedure requires to determine the acquisition point number which is closest to the zero delay between the two pump pulses and the phase associated with it. The method is well described in an article by Helbing and Hamm. ${ }^{36}$ We start with the interferogram between the two pump pulses displayed in Fig. 6(a). We then cut the beginning of the interferogram until its Fourier transform exhibits a flat phase. In the case depicted in Fig. 6(a), the flattest phase (Fig. 6(b)) is found when cutting at point 317 the interferogram. We will then remove the first 316 points of data before applying the Fourier transform. As we measure the interferogram between pump pulses during the 2DES experiment, we can calculate the phase spectrum for each data set and use this phase to correct the $2 \mathrm{D}$ spectrum properly.

\section{Dispersion compensation}

Compensation of the dispersion introduced by TWINS is important as any residual chirp can distort the $2 \mathrm{D}$ spectra, ${ }^{48}$ and because it allows to perform 2DES with the best time resolution afforded by the broadband transform-limited (TL)
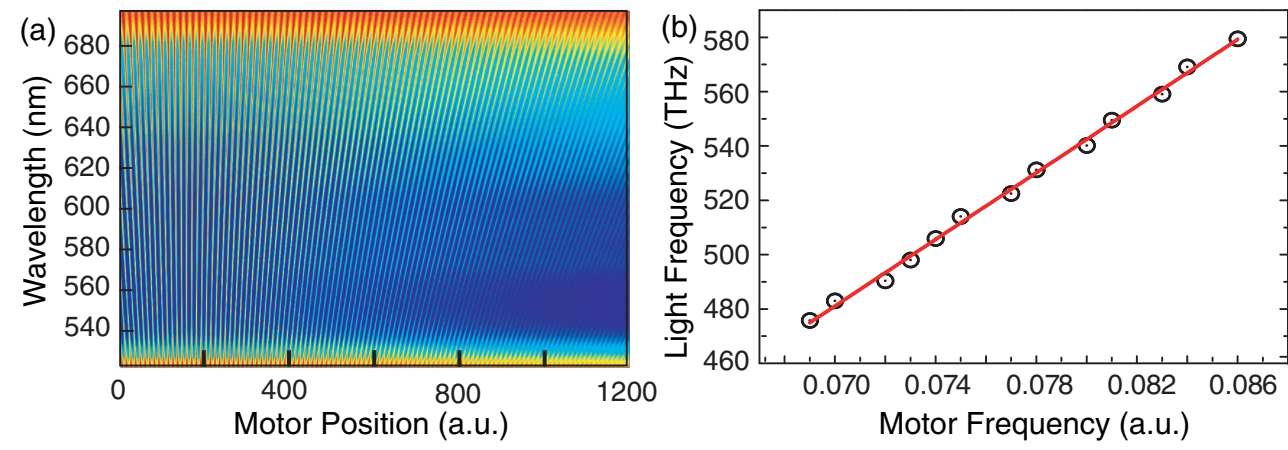

FIG. 5. (a) Frequency-resolved linear autocorrelation of the two pump pulses during continuous scan. (b) Calibration curve obtained from (a). 

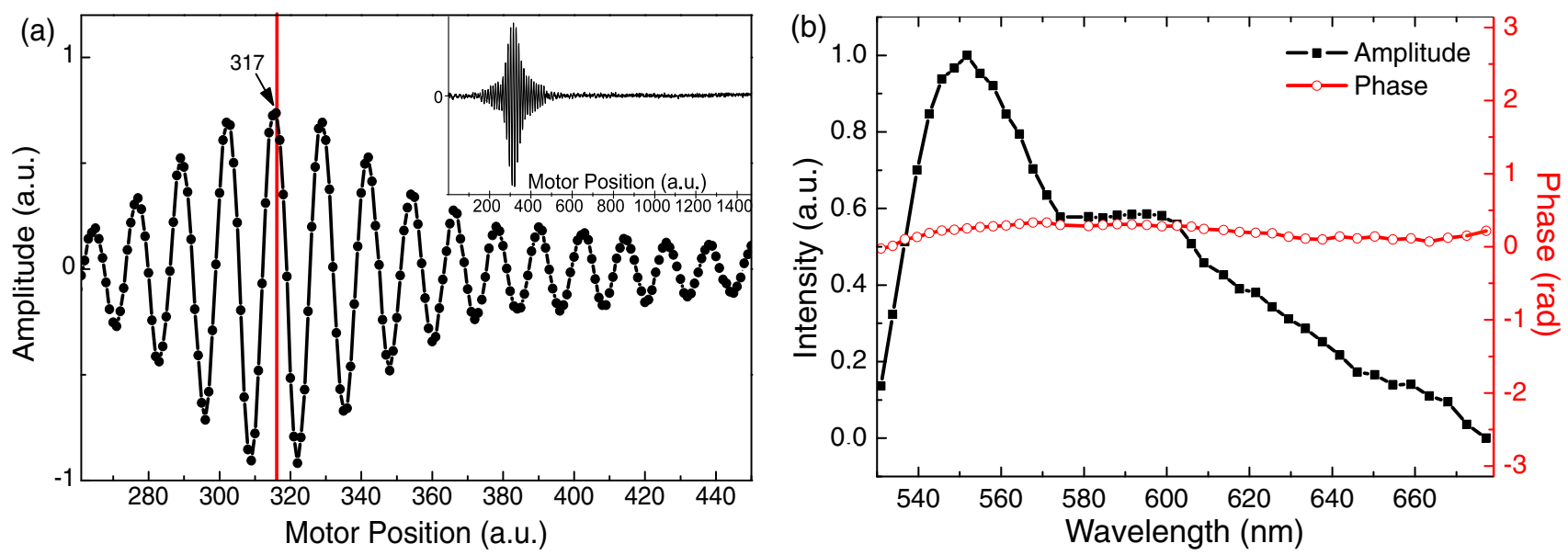

FIG. 6. (a) zoom of the interferogram between the two pump pulses measured on a single element detector (inset: full interferogram). (b) Amplitude and phase of the Fourier transform interferogram after phasing.

pulses produced by our NOPAs. The probe beam preserves the input duration, as it does not cross any dispersive optics before the sample. We measure the probe pulse duration at the sample position using the frequency-resolved optical gating (FROG) technique. ${ }^{49}$ In contrast, the pump pulses cross a large amount of glass, with two polarizers, four $\alpha$ BBO wedges and one $\alpha$-BBO plate, introducing a group delay dispersion (GDD) in the visible of more than $500 \mathrm{fs}^{2}$. This GDD can be compensated with a second pair of DCMs to restore nearly TL pulses at the sample. To monitor the dispersion in the pump beam, we used a variation of spectral interferometry, ${ }^{50}$ the so called- Spatial Encoded Arrangement for Temporal Analysis by Dispersing a Pair of Light E-fields (SEA-TADPOLE) ${ }^{51,52}$ We measure the spatial interferences pattern between the probe and pump pulses at the sample position, which allows direct monitoring of the spectral phase difference between the two beams. We can first minimize dispersion by varying the number of bounces on the DCMs. Because the number of bounces has to be even (DCMs are designed to work in pairs), the GDD can be controlled only to a precision of $\approx 50 \mathrm{fs}^{2}$. To finely tune further the dispersion, we can optimize the insertion of wedge 4 (static) of the TWINS block $\mathrm{C}$ and thus cancel the residual GDD on the bandwidth of interest (see Fig. 7). To illustrate the efficiency of the chirped mirrors, we also plot the spectral phase difference introduced by the TWINS and the DCMs alone. As can be seen, the dispersion introduced by TWINS can be completely compensated, ensuring pulses lengths as close as possible to the TL value. It is also possible to decrease the insertion of the wedges of block B in Fig. 3(a) into the beam in order to reduce the GDD of TWINS, and then reduce the number of bounces on the DCMs, but this is done at the cost of smaller accessible $t_{1}$, i.e., lower resolution of the pump axis in 2DES maps.

After the TWINS, and for large $t_{1}$ delays, the two pump pulses cannot be considered as exact replicas because they cross materials with different dispersion. The second pump pulse (pulse 2) is static during a $t_{1}$ scan, and keeps its original TL duration ensured by the proper dispersion compensation previously described. This is not the case for the first pump pulse, for which ordinary index material is replaced by extraordinary index material during a $\mathrm{t}_{1}$ scan. Consequently, pulse 1 is affected by a different dispersion that can be calculated by considering the path-length in the wedges and the difference between the group velocity dispersion (GVD) for the ordinary (GVDo) and extraordinary (GVDe) polarizations, which is reported in Table I.

In Table II, we present the effect of dispersion on a 10 fs pulse for different center wavelengths and different $t_{1}$ delays. For each value of $t_{1}$, we also added the corresponding relative spectral resolution $(\Delta \lambda / \lambda)$ of the pump axis of the $2 \mathrm{D}$ map. We observe that the effect is small and even negligible for most of the cases presented in this table. In the ultraviolet and for long $t_{1}$ delays, the effect is the strongest, as the output pulse length is more than twice the input pulse length. However, this is an extreme case corresponding to a very high spectral resolution $(\Delta \lambda / \lambda=0.003)$, which is not useful practically. Already a resolution of $\Delta \lambda / \lambda=0.02$ at $300 \mathrm{~nm}$ is more than enough for most of the samples of interest in this

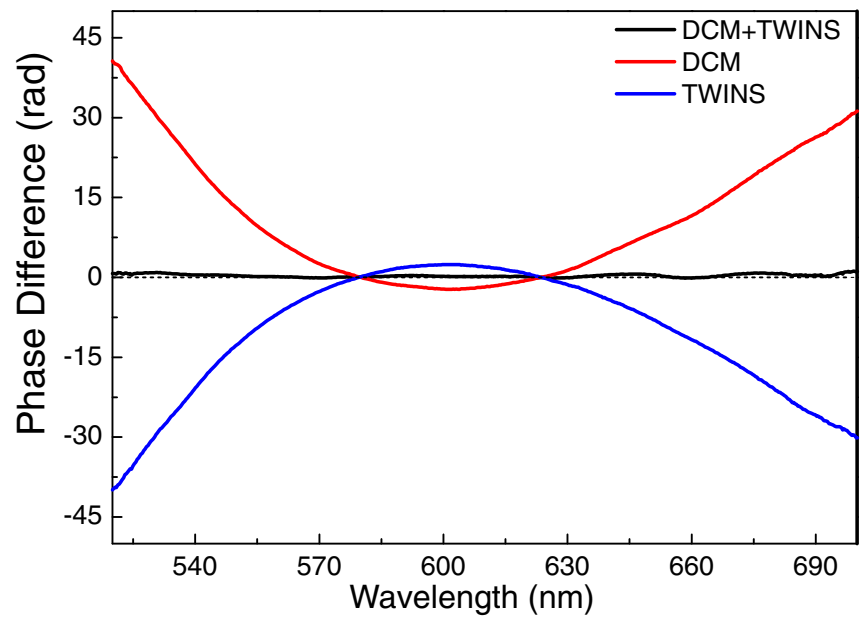

FIG. 7. SEA-TADPOLE measurement of the spectral phase difference between pump and probe beams. SEA Tadpole, pump VS probe. Black, dispersion curve with TWINS and DCM in the pump beam. Blue, only TWINS is in the pump beam. Red, only DCMs are in the pump beam. 
TABLE II. Output pulsewidth (in fs) and spectral resolution ( $\Delta \lambda / \lambda$ in parenthesis) corresponding to a $10 \mathrm{fs}$ input pulsewidth for different $\mathrm{t}_{1}$ values, at different central wavelengths.

\begin{tabular}{lllccc}
\hline \hline $\mathrm{t}_{1} \backslash \lambda$ & \multicolumn{1}{c}{$1100 \mathrm{~nm}$} & $900 \mathrm{~nm}$ & $700 \mathrm{~nm}$ & $500 \mathrm{~nm}$ & $300 \mathrm{~nm}$ \\
\hline $50 \mathrm{fs}$ & $10.30(0.072)$ & $10.03(0.06)$ & $10.05(0.047)$ & $10.10(0.032)$ & $10.36(0.02)$ \\
$100 \mathrm{fs}$ & $10.1(0.036)$ & $10.13(0.03)$ & $10.19(0.023)$ & $10.38(0.016)$ & $11.36(0.01)$ \\
$200 \mathrm{fs}$ & $10.42(0.018)$ & $10.5(0.015)$ & $10.75(0.012)$ & $11.45(0.008)$ & $14.71(0.005)$ \\
$400 \mathrm{fs}$ & $11.59(0.009)$ & $11.88(0.008)$ & $12.72(0.006)$ & $14.98(0.004)$ & $23.78(0.003)$ \\
\hline \hline
\end{tabular}

wavelength range, and we observe that the effect of dispersion is indeed very small in that case.

\section{Scattering suppression}

Signals coming from the scattering of the pump beam are usually an issue in 2D spectroscopy, because the interference of the scattered light with the probe light depends on delay $t_{2}$. These spurious signals appear on the diagonal of a 2D spectrum and can in some cases exceed the nonlinear signal of interest. Many strategies have been used to minimize or completely eliminate scattering signals in $2 \mathrm{D}$ spectroscopy. $4,23,28,35,38,53,54$ They all rely on the idea of phase cycling or quasi-phase cycling, which uses the fact that, for a given wavelength $\lambda$, the interference signal between pump and probe is opposite if the phase of one of the pulse changes by $\pi$ (or the delay between them is varied by $\frac{\lambda}{2}$ for quasi-phase cycling). If one averages two signals with $t_{2}$ varied by $\frac{\lambda}{2}$, the scatter will be eliminated at $\lambda$ while the interesting signal will remain unchanged for such a small delay change. With this approach scattering is suppressed to the first order, i.e., around the central wavelength $\lambda$. Scatter can also be suppressed to higher orders by averaging several signals at different $t_{2}$ delays, ${ }^{36}$ but this procedure can alter the time resolution of the experiment for few-cycle pulses. The best way to remove scattering signal with quasi-phase cycling is to modulate the phase between pump and probe at the repetition rate of the laser, thus making best use of the phase correlation between successive pulses. ${ }^{35,54}$

Here we use a very simple method that allows to modulate the delay $t_{2}$ on a pulse to pulse basis. Because we use a chopper at $500 \mathrm{~Hz}$, we need to modulate the signal at $250 \mathrm{~Hz}$. This is done by attaching a loudspeaker $(0.3 \mathrm{~W}, 8 \Omega)$ to a mirror mount on the probe beam. We feed the loudspeaker with a sine waveform which is synchronized to the laser repetition rate. The sound wave makes a small vibration on the mirror that can be tuned precisely to the amplitude of $\frac{\lambda}{2}$ in the visible range. We can easily tune the amplitude and phase of the vibration to center the scattering suppression to the desired wavelength. Fig. 8 shows the scattering signal obtained by focusing both pump and probe pulses on a small pinhole. Without modulation (black solid line the figure), the scattering signal has an amplitude of a few percent. We see that we can reduce significantly this scatter in the wavelength range of interest by turning on the loudspeaker (see red line in Fig. 8). While we could perform only first order modulation with our loudspeaker, it is possible to increase the modulation amplitude to eliminate the scatter to higher order.

\section{E. Continuous scanning of coherence time}

Continuous scanning of $t_{1}$ in time-domain 2D spectroscopy is a useful strategy for improving data collection efficiency. ${ }^{36,55}$ The idea is to measure the full range of coherence times as quickly as possible, but still below the Nyquist sampling rate (corresponding to 2 points per fringe), to make best use of pulse to pulse correlation, and eliminate the noise introduced by slow power fluctuations of the laser source. In our case, the maximum speed of the motor of $2 \mathrm{~mm} / \mathrm{s}$ can only allow a speed of about a fifth of the Nyquist rate.

Continuously scanning the coherence time does not require a lot of effort in our case, as it does not need any kind of position tracking. In fact, a constant velocity of the TWINS motor is sufficient to acquire time-domain data in a reproducible way, ensuring equally spaced time points during acquisition. The motor movement is not synchronized with the $1 \mathrm{kHz}$ repetition rate of the laser. This implies that we do not reproduce exactly the same positions at every scan. There is a time jitter that cannot exceed $1 \mathrm{~ms}$ between the position of the motor and the pulse arrival. At the scan rate that we use, which gives approximately 10 points per fringe of the visible radiation, this is equivalent to binning the time data with a bin size of $\lambda / 10$. Even if software or electronic delays shift in time the acquisition relative to the motor movement, the different scans can be overlapped as we record, in parallel to the 2D signal, the pump pulse pair interferogram on a single element photodiode after sample (PD in Fig. 4). Fig. 9 displays different interferograms for subsequent scans. As can be seen, the average over all scans is very similar to each individual scan, confirming the reliability of the averaging procedure. The

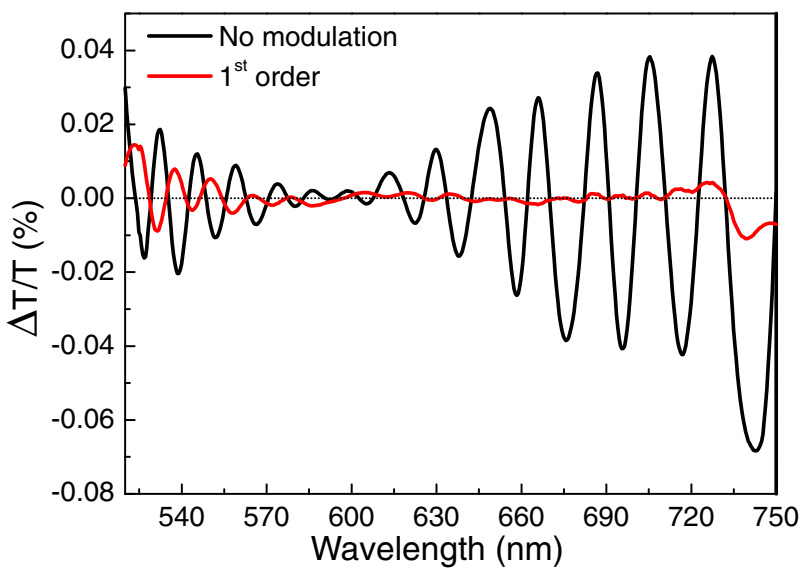

FIG. 8. Scattering spectrum with and without $\pm \frac{\lambda}{2}$ modulation of delay between pump and probe. 


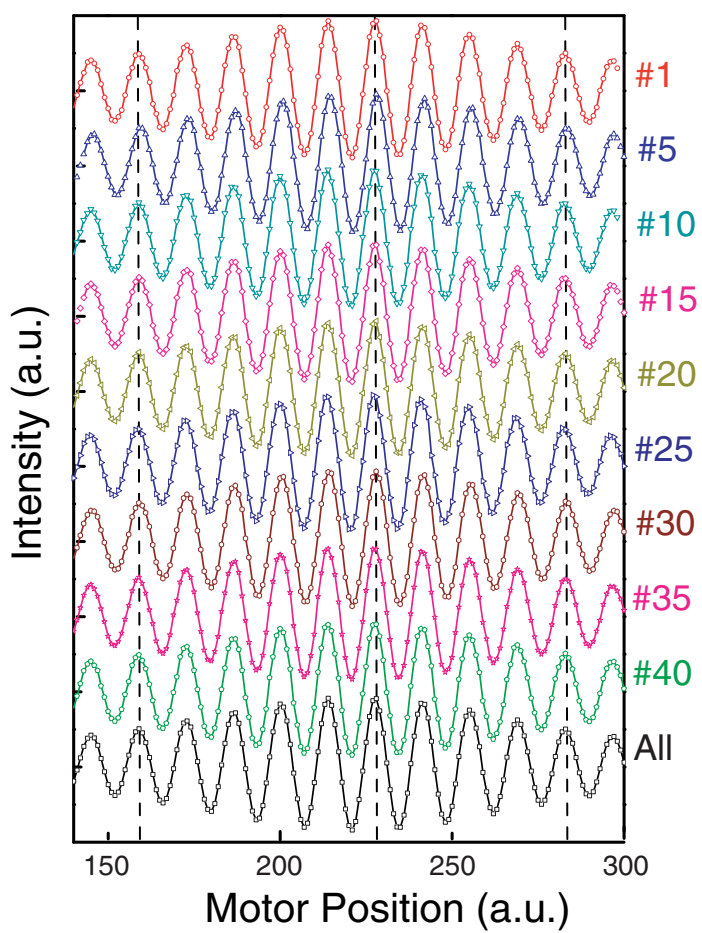

FIG. 9. Interferogram measured for different scan number indicated on the right. The lower one is the average of 40 scans.

interferograms are also used to overlap forward and backward scans, and to calculate the phase to correct the 2D spectra. The bin size thus depends on the speed of the motor, which means that if we want to scan faster, a kind of delay tracking will be necessary during the acquisition. The motor controller could be in principle used for this purpose, but it is currently not possible to update the position from the motor encoder at $\mathrm{kHz}$ rate. This could be achieved by a synchronized external counter reading continuously triggers sent from the motor controller. We will consider this possibility for future upgrade of the setup with a faster motor.

In the fast scanning mode, we set the parameters of the acquisition board for a maximum value of the $t_{1}$ time (e.g., $100 \mathrm{fs}$ ). We calculate the required scan distance to achieve with the motor and the number of fringes to which it corresponds at the central wavelength of the pulses. We then calculate the number of points to measure (10 times number of fringes) and deduce the motor speed to achieve the delay during the given time. Immediately after launching the motor to its final position, we start the acquisition of the probe pulse spectra. The data points acquired this way during the movement of the motor are equally spaced as the motor speed is linear and reproducible. The measurement of the interferogram between the two pump pulses while scanning permits to overlap precisely forward and backward scans. A routine checks the proper overlap of the backward and forward interferograms to correct for a possible shift between the two scans. In practice, this routine shifts by one or two points the interferograms, but not more. These shifts are due to software or electronic delays, and to the fact that the motor is not synchronized with the laser triggers. We repeat the same procedure for every $t_{2}$ delay, and average the data for successive

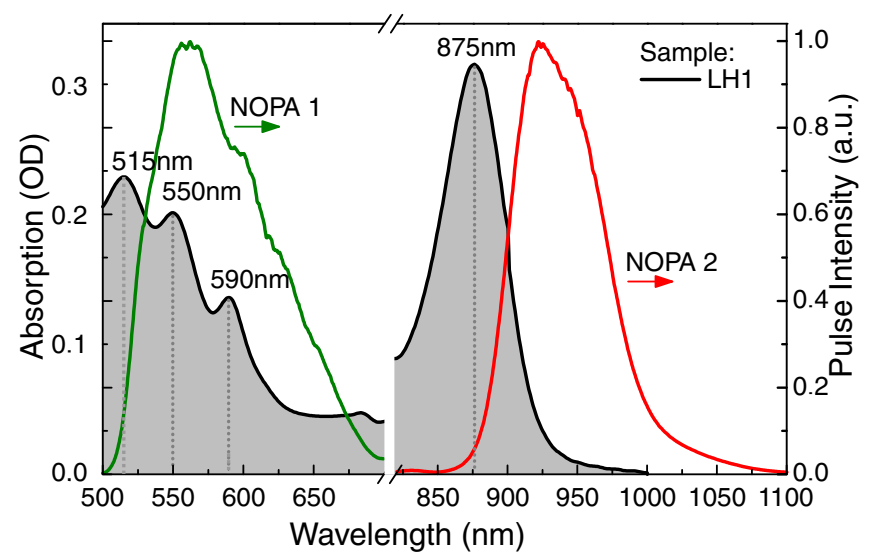

FIG. 10. Black line: Absorption spectrum of LH 1. Green and red: spectrum of NOPA 1 and NOPA 2, respectively.

$\mathrm{t}_{2}$ scans until the 2D spectra displayed live during the experiment exhibit a satisfactory signal to noise ratio.

\section{RESULTS}

To assess the performance of our broadband 2DES spectrometer, we performed experiments on the light harvesting complex 1 (LH1) of the purple bacterium Rhodospirillum rubrum. Samples of LH1 were prepared as reported in Ref. 56, and suspended in a buffer solution contained in a $200-\mu \mathrm{m}$-thick cell. The linear absorption spectrum of LH1 is shown as a solid black line in Fig. 10. One can recognize at $550 \mathrm{~nm}$ the peak corresponding to the $S_{0} \rightarrow S_{2}$ transition of the carotenoid (Car) spirilloxanthin, and its vibronic replica at 550 and $515 \mathrm{~nm}$. The peaks at 875 and $590 \mathrm{~nm}$ correspond, respectively, to the $\mathrm{Q}_{y}$ and $\mathrm{Q}_{x}$ transitions of the bacteriochlorophyll (BChl) B875. The spectra of the visible and near-IR pulses used for degenerate and two-colour 2DES are shown in Fig. 10 in green and red lines, respectively.

Figure 11 shows a sequence of degenerate and twocolor 2DES spectra for different $t_{2}$ delays. At early times $\left(t_{2}\right.$ $=40 \mathrm{fs}$ ), in the degenerate 2D spectrum (Fig. 11(a), left column), we observe positive red peaks corresponding to the photobleaching (PB) of the $\mathrm{S}_{0} \rightarrow \mathrm{S}_{2}$ transition of the Car (peak A) and of the $\mathrm{Q}_{x}$ transition of the BChl (peak B). We also see cross peaks due to the stimulated emission (SE) from $S_{2}$ to $S_{0}$ in the Car (peaks C). Already at $t_{2}=120 \mathrm{fs}$ (Fig. 11(b)) the 2DES spectra change dramatically due to the ultrafast $\mathrm{S}_{2} \rightarrow \mathrm{S}_{1}$ internal conversion in the Car; we observe the build-up of two strong negative cross-peaks, corresponding to photoinduced absorption (PA) from the $S_{1}$ state (peak D) and from the so-called $S^{*}$ state (peak E) of the Car. ${ }^{56,57}$ This formation is completed within $300 \mathrm{fs}$ and at $\mathrm{t}_{2}=1 \mathrm{ps}$ we observe the cross-peaks narrowing due to vibrational relaxation within $S_{1}$. The right-hand side of Fig. 11 shows a sequence of two-colour 2DES spectra, pumping in the visible and probing in the NIR, in the region of the $\mathrm{Q}_{y}$ transition of the BChl. We see at early times $\left(t_{2}=40\right.$ fs, Fig. 11(a)) two distinct cross-peaks corresponding to the coupling between $\mathrm{Q}_{x}$ and $\mathrm{Q}_{y}$ transitions in the BChl (peak $\mathrm{F}$ ) and to the energy transfer between Car and BChl (peak G). We also observe the 

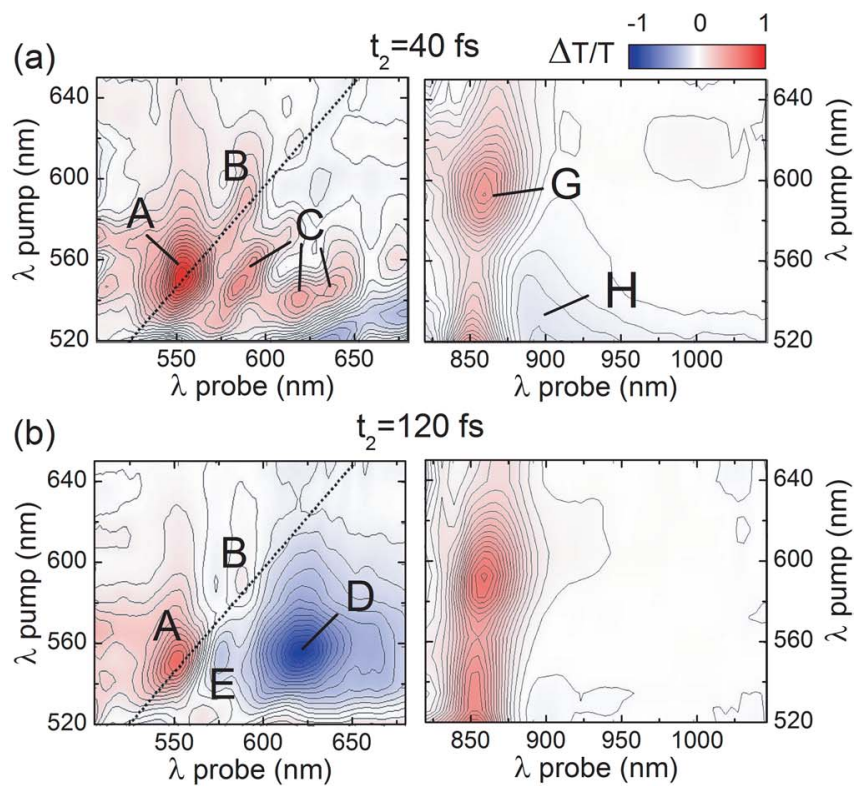

$=120 \mathrm{fs}$
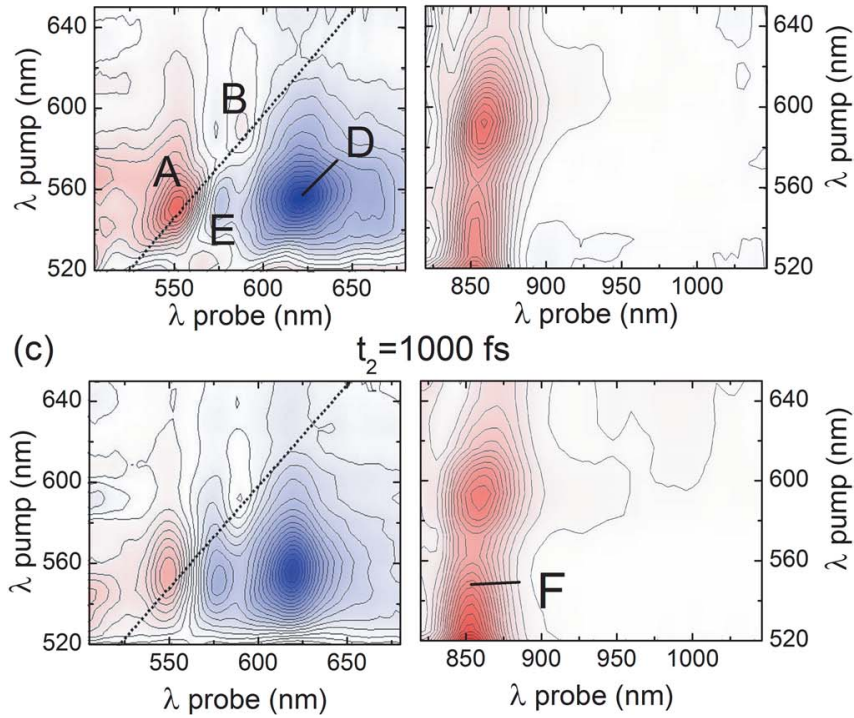

$\mathrm{t}_{2}=1000 \mathrm{fs}$

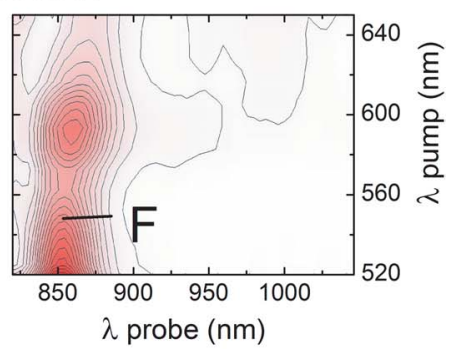

FIG. 11. (a)-(c): 2D spectra of the LH1 complex of purple bacterium rhodospirillum rubrum for different values of $t_{2}$ delays. Left column: degenerate 2D spectra in the visible. Right column: 2D 2-color visible pump/infrared probe spectra.

short-lived excited state absorption of the $\mathrm{S}_{2} \rightarrow \mathrm{S}_{n}$ of the Car (peak $\mathrm{H})$. The two cross-peaks grow in time with different dynamics, enabling us to disentangle the $\mathrm{Q}_{x} \rightarrow \mathrm{Q}_{y}$ internal conversion process in the $\mathrm{BChl}$ from the energy transfer between Car and BChl. We believe that these data demonstrate the unprecedented capability of our system to perform two-colour 2DES with very broad spectral coverage and with minimal experimental effort. Our results clearly show that the setup allows easy recording of $2 \mathrm{D}$ spectra with high time resolution and flexibility in the probe window. Different polarization schemes are also straightforward to implement, for example, it is easy to remove the polarizer at the output of the TWINS and produce perpendicular pump pulses, allowing to perform polarization schemes with enhanced sensitivity. ${ }^{32,33,58}$

\section{CONCLUSION}

In this paper, we presented a 2DES setup based on birefringent wedges which is suitable for routine measurements of 2D spectra in the visible to the NIR. The coherence time can be scanned continuously without any need of delay tracking, thanks to the high accuracy of the TWINS device, simplifying considerably the setup. We demonstrated the reliability of the method by measuring 2D spectra of the LH1 complex of a purple bacterium and highlighting the interactions between carotenoids and bacteriochlorophylls. We demonstrated easy scattering suppression with a loudspeaker. The extension to the UV range, where the phase determination is even more challenging due to the short wavelength, is straightforward and will allow to study $\mathrm{DNA}^{59,60}$ and protein ${ }^{17,22}$ photophysics. We are confident that our approach, because of its simplicity and the minimal experimental effort to implement it in a pump-probe experiment, will motivate a broad nonspecialist community of physicists, chemists and biologists to move towards multidimensional spectroscopy.

\section{ACKNOWLEDGMENTS}

We thank Professor Cogdell for the LH 1 samples. We would like to acknowledge Alice Cantaluppi and Giulio Maria Rossi for building NOPAs and the first version of the setup.

This work was supported by the European Research Council Advanced Grant STRATUS (ERC-2011-AdG No. 291198). J.R. thanks the Swiss National Science Foundation for financial support (Fellowship PBZHP2_143444).

${ }^{1}$ J. P. Likforman, M. Joffre, and V. Thierry-Mieg, Opt. Lett. 22, 1104-1106 (1997).

${ }^{2}$ D. M. Jonas, Science 300, 1515-1517 (2003).

${ }^{3}$ J. D. Hybl, A. A. Ferro, and D. M. Jonas, J. Chem. Phys. 115, 6606-6622 (2001).

${ }^{4}$ P. Tian, D. Keusters, Y. Suzaki, and W. S. Warren, Science 300, 1553-1555 (2003).

${ }^{5}$ R. M. Hochstrasser, Proc. Natl. Acad. Sci. U.S.A. 104, 14190-14196 (2007).

${ }^{6}$ M. Cho, Two-Dimensional Optical Spectroscopy (CRC Press, 2009).

${ }^{7}$ P. Hamm and M. T. Zanni, Concepts and Methods of $2 D$ Infrared Spectroscopy (Cambridge University Press, 2011).

${ }^{8}$ T. Brixner, J. Stenger, H. M. Vaswani, M. Cho, R. E. Blankenship, and G. R. Fleming, Nature (London) 434, 625-628 (2005).

${ }^{9}$ N. Christensson, F. Milota, A. Nemeth, J. Sperling, H. F. Kauffmann, T. Pullerits, and J. Hauer, J. Phys. Chem. B 113, 16409-16419 (2009).

${ }^{10}$ P. D. Dahlberg, A. F. Fidler, J. R. Caram, P. D. Long, and G. S. Engel, J. Phys. Chem. Lett. 4, 3636-3640 (2013).

${ }^{11}$ E. De Re, G. Schlau-Cohen, V. Huxter, R. Leverenz, R. Mathies, and G. Fleming, EPJ Web Conf. 41, 08008 (2013).

${ }^{12}$ F. Milota, J. Sperling, A. Nemeth, D. Abramavicius, S. Mukamel, and H. F. Kauffmann, J. Chem. Phys. 131, 054510 (2009).

${ }^{13}$ F. Milota, J. Sperling, A. Nemeth, and H. F. Kauffmann, Chem. Phys. 357, 45-53 (2009).

${ }^{14}$ S. Cundiff, A. Bristow, M. Siemens, H. Li, G. Moody, D. Karaiskaj, X. Dai, and T. Zhang, IEEE J. Select. Topics Quantum Electron. 18(1), 318328 (2012).

${ }^{15}$ M. W. Graham, T. R. Calhoun, A. A. Green, M. C. Hersam, and G. R. Fleming, Nano Lett. 12, 813-819 (2012).

${ }^{16}$ D. B. Turner, P. Wen, D. H. Arias, K. A. Nelson, H. Li, G. Moody, M. E. Siemens, and S. T. Cundiff, Phys. Rev. B 85, 201303 (2012).

${ }^{17}$ J. Jiang, D. Abramavicius, C. Falvo, B. M. Bulheller, J. D. Hirst, and S. Mukamel, J. Phys. Chem. B 114, 12150-12156 (2010).

${ }^{18}$ B. A. West, J. M. Womick, and A. M. Moran, J. Phys. Chem. A 115, 86308637 (2011).

${ }^{19}$ G. Auböck, C. Consani, F. van Mourik, and M. Chergui, Opt. Lett. 37, 2337-2339 (2012).

${ }^{20}$ P. Hamm, M. Lim, and R. M. Hochstrasser, J. Phys. Chem. B 102, 61236138 (1998).

${ }^{21}$ V. P. Singh, A. F. Fidler, B. S. Rolcynski, and G. S. Engel, J. Chem. Phys. 139, 084201 (2013).

${ }^{22}$ C. Consani, G. Auböck, F. v. Mourik, and M. Chergui, Science 339, 15861589 (2013).

${ }^{23}$ W. P. de Boeij, M. S. Pshenichnikov, and D. A. Wiersma, Chem. Phys. Lett. 238, 1-8 (1995).

${ }^{24}$ T. Brixner, T. Manal, I. V. Stiopkin, and G. R. Fleming, J. Chem. Phys. 121, 4221-4236 (2004). 
${ }^{25}$ M. L. Cowan, J. P. Ogilvie, and R. J. D. Miller, Chem. Phys. Lett. 386, 184-189 (2004).

${ }^{26}$ L. P. DeFlores, R. A. Nicodemus, and A. Tokmakoff, Opt. Lett. 32(20), 2966 (2007)

${ }^{27}$ S.-H. Shim, D. B. Strasfeld, Y. L. Ling, and M. T. Zanni, Proc. Natl. Acad. Sci. U.S.A. 104, 14197-14202 (2007).

${ }^{28}$ E. M. Grumstrup, S.-H. Shim, M. A. Montgomery, N. H. Damrauer, and M. T. Zanni, Opt. Exp. 15, 16681-16689 (2007).

${ }^{29}$ A. D. Bristow, D. Karaiskaj, X. Dai, T. Zhang, C. Carlsson, K. R. Hagen, R. Jimenez, and S. T. Cundiff, Rev. Sci. Instrum. 80, 073108 (2009).

${ }^{30}$ T. Zhang, C. N. Borca, X. Li, and S. T. Cundiff, Opt. Exp. 13(19), 7432 (2005).

${ }^{31}$ U. Selig, F. Langhojer, F. Dimler, T. Löhrig, C. Schwarz, B. Gieseking, and T. Brixner, Opt. Lett. 33, 2851-2853 (2008).

${ }^{32}$ M. T. Zanni, N.-H. Ge, Y. S. Kim, and R. M. Hochstrasser, Proc. Natl. Acad. Sci. U.S.A. 98, 11265-11270 (2001).

${ }^{33}$ J. Réhault and J. Helbing, Opt. Exp. 20, 21665-21677 (2012).

${ }^{34}$ J. Réhault and J. Helbing, EPJ Web Conf. 41, 05003 (2013).

${ }^{35}$ S.-H. Shim and M. T. Zanni, Phys. Chem. Chem. Phys. 11, 748-761 (2009).

${ }^{36}$ J. Helbing and P. Hamm, J. Opt. Soc. Am. B 28, 171-178 (2011).

${ }^{37}$ D. B. Turner, K. W. Stone, K. Gundogdu, and K. A. Nelson, Rev. Sci. Instrum. 82, 081301-1-081301-22 (2011).

${ }^{38}$ J. A. Myers, K. L. Lewis, P. F. Tekavec, and J. P. Ogilvie, Opt. Exp. 16, 17420-17428 (2008)

${ }^{39}$ D. Brida, C. Manzoni, and G. Cerullo, Opt. Lett. 37, 3027-3029 (2012).

${ }^{40}$ J. Réhault, M. Maiuri, C. Manzoni, D. Brida, J. Helbing, and G. Cerullo, Opt. Exp. 22, 9063 (2014).

${ }^{41}$ M. U. Wehner, M. H. Ulm, and M. Wegener, Opt. Lett. 22, 1455-1457 (1997).

${ }^{42}$ S. P. Davis, M. C. Abrams, and J. W. Brault, Fourier Transform Spectrometry (Academic Press, 2001)

${ }^{43}$ M. Zavelani-Rossi, G. Cerullo, S. De Silvestri, L. Gallmann, N. Matuschek, G. Steinmeyer, U. Keller, G. Angelow, V. Scheuer, and T. Tschudi, Opt. Lett. 26, 1155-1157 (2001).
${ }^{44}$ C. Manzoni, D. Polli, and G. Cerullo, Rev. Sci. Instrum. 77, 023103 (2006).

${ }^{45}$ G. Cerullo, C. Manzoni, L. Lüer, and D. Polli, Photochem. Photobiol. Sci. 6, 135-144 (2007).

${ }^{46}$ A. M. Siddiqui, G. Cirmi, D. Brida, F. X. Kärtner, and G. Cerullo, Opt. Lett. 34, 3592-3594 (2009).

${ }^{47}$ D. Polli, L. Lüer, and G. Cerullo, Rev. Sci. Instrum. 78, 103108 (2007).

${ }^{48}$ P. F. Tekavec, J. A. Myers, K. L. M. Lewis, F. D. Fuller, and J. P. Ogilvie, Opt. Exp. 18, 11015-11024 (2010).

${ }^{49}$ R. Trebino, Frequency-Resolved Optical Gating: The Measurement of Ultrashort Laser Pulses: The Measurement of Ultrashort Laser Pulses (Springer, 2000).

${ }^{50}$ L. Lepetit, G. Chériaux, and M. Joffre, J. Opt. Soc. Am. B 12, 2467-2474 (1995).

${ }^{51}$ D. N. Fittinghoff, I. A. Walmsley, J. L. Bowie, J. N. Sweetser, R. T. Jennings, M. A. Krumbügel, K. W. DeLong, and R. Trebino, Opt. Lett. 21, 884-886 (1996).

${ }^{52}$ P. Bowlan, P. Gabolde, A. Shreenath, K. McGresham, R. Trebino, and S. Akturk, Opt. Exp. 14, 11892-11900 (2006).

${ }^{53}$ A. W. Albrecht, J. D. Hybl, S. M. G. Faeder, and D. M. Jonas, J. Chem. Phys. 111, 10934-10956 (1999).

${ }^{54}$ R. Bloem, S. Garrett-Roe, H. Strzalka, P. Hamm, and P. Donaldson, Opt. Exp. 18, 27067-27078 (2010).

${ }^{55} \mathrm{~S}$. T. Roberts, J. J. Loparo, K. Ramasesha, and A. Tokmakoff, Opt. Commun. 284, 1062-1066 (2011).

${ }^{56}$ C. C. Gradinaru, J. T. M. Kennis, E. Papagiannakis, I. H. M. v. Stokkum, R. J. Cogdell, G. R. Fleming, R. A. Niederman, and R. v. Grondelle, Proc. Natl. Acad. Sci. U.S.A. 98, 2364-2369 (2001).

${ }^{57}$ E. Papagiannakis, J. T. M. Kennis, I. H. M. v. Stokkum, R. J. Cogdell, and R. v. Grondelle, Proc. Natl. Acad. Sci. U.S.A. 99, 6017-6022 (2002).

${ }^{58}$ J. Réhault, V. Zanirato, M. Olivucci, and J. Helbing, J. Chem. Phys. 134, 124516-1-124516-10 (2011).

${ }^{59}$ B. A. West and A. M. Moran, J. Phys. Chem. Lett. 3, 2575-2581 (2012).

${ }^{60}$ B. A. West, P. G. Giokas, B. P. Molesky, A. D. Ross, and A. M. Moran, Opt. Exp. 21, 2118-2125 (2013). 